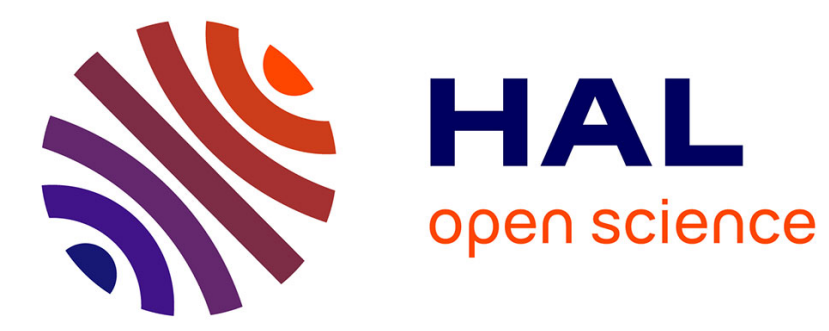

\title{
Interpretation of Finite Volume discretization schemes for the Fokker Planck equation as gradient flows for the discrete Wasserstein distance
}

F Al Reda, B Maury

\section{To cite this version:}

F Al Reda, B Maury. Interpretation of Finite Volume discretization schemes for the Fokker Planck equation as gradient flows for the discrete Wasserstein distance. 2016. hal-01288983

\section{HAL Id: hal-01288983 \\ https://hal.science/hal-01288983}

Preprint submitted on 15 Mar 2016

HAL is a multi-disciplinary open access archive for the deposit and dissemination of scientific research documents, whether they are published or not. The documents may come from teaching and research institutions in France or abroad, or from public or private research centers.
L'archive ouverte pluridisciplinaire HAL, est destinée au dépôt et à la diffusion de documents scientifiques de niveau recherche, publiés ou non, émanant des établissements d'enseignement et de recherche français ou étrangers, des laboratoires publics ou privés. 


\title{
F. Al Reda and B. Maury
}

\section{Interpretation of Finite Volume discretization schemes for the Fokker Planck equation as gradient flows for the discrete Wasserstein distance}

\begin{abstract}
This paper establishes a link between some space discretization strategies of the Finite Volume type for the Fokker-Planck equation in general meshes (Voronoï tesselations) and gradient flows on the underlying networks of cells, in the framework of discrete Wasserstein distances on graphs recently proposed by Maas [6].
\end{abstract}

Keywords: Gradient flows, Wasserstein distance, Finites Volumes discretization, Fokker-Planck equation, Heat equation, Resistive Network, Markov Kernel.

AMS Subject Classification: 60J60, 94C15, 35K05, 35K08, 65N08, 60J27, $60 \mathrm{~J} 28$.

\section{Introduction}

We aim here at identifying gradient flow structures in some space-discretization schemes of the Fokker-Planck equation on general meshes, in the spirit of the approaches proposed recently in [1,2] for cartesian discretizations. Since the core of the paper consists in building links between macroscopic notions / properties and their discrete counterparts, in a context where two reference measures are present (uniform Lebesgue measure and stationary measure associated to an attractive potential), let us start by fixing some principles in terms of notation. Probability measures will be denoted by the letter $p$ (we shall use the same letter to denote their density with respect to the underlying Lebesgue measure, or its discrete counterpart), stationary measures (with respect to some evolution process) by $\pi$, and relative densities with respect to $\pi$ by $\rho$. All discrete notions

F. Al Reda: Laboratoire de Mathématiques d'Orsay, Université Paris-Sud, 91405 Orsay cedex, France

B. Maury: Laboratoire de Mathématiques d'Orsay, Université Paris-Sud, 91405 Orsay cedex, France 
will be singled out by a tilda sign, e.g. $\tilde{p}, \tilde{\pi}$, etc ... The space variable will be denoted by $r$, while $x$ and $y$ will be used to denote discrete vertices.

Since the seminal work of Jordan, Kinderlehrer and Otto [3] in 1998, it is known that the Fokker-Planck (FP) equation in a domain $\Omega$ :

$$
\partial_{t} p-\Delta p-\nabla \cdot(p \nabla \Phi)=0
$$

with appropriate no-flux boundary conditions, can be interpreted in the Wasserstein space as the gradient flow for

$$
H(p)=\int_{\Omega} p \log \left(\frac{p}{\pi}\right) \mathrm{d} r \quad\left(=\int_{\Omega} \rho \log (\rho) \mathrm{d} \pi \text { with } \rho=p / \pi\right),
$$

that is the relative entropy with respect to the stationary measure $\pi=e^{-\Phi}$, up to a normalization constant. This property is schematized in the diagram below (see Fig. 1, blocks $A-B-C$, on the top), and we refer the reader to $[4,5]$ for a thorough description of the underlying theory.

At the discrete level, a similar framework has been proposed in $[6,7]$. The euclidean domain is replaced by a network $\mathcal{N}$, defined by its (finite) set of vertices $V$ and a Markovian kernel

$$
(K(x, y))_{x, y \in V}, \text { with } K(x, y) \geq 0, \sum_{y \in V} K(x, y)=1 \quad \forall x \in V .
$$

The stationary measure is denoted by $\tilde{\pi}$, it verifies $\widetilde{\pi}={ }^{t} K \widetilde{\pi}$. It is unique as soon as $K$ is irreducible, i.e. $\forall x, y \in V$ there exists a path $\left(x_{0}=x, x_{1}, x_{2}, \ldots, x_{m}=y\right)$ such that $K\left(x, x_{1}\right) \times K\left(x_{1}, x_{2}\right) \times \ldots \times K\left(x_{m-1}, y\right)>0$, and then $\tilde{\pi}(x)>0$ for all $x \in V$. We say that $K$ is reversible if $\tilde{\pi}(x) K(x, y)=K(y, x) \widetilde{\pi}(y)$ for all $x, y$ in $V$ (detailed balance equation). The discrete counterpart of the FP equation is the heat flow equation

$$
\partial_{t} \widetilde{\rho}+(I-K) \widetilde{\rho}=0,
$$

where $\widetilde{\rho}$ is the density of a probability measure $\widetilde{p}$ on $V$ with respect to $\tilde{\pi}$. Note that the straight discrete counterpart of FP equation would be an equation of the measure $\tilde{p}$ itself, with $K$ replaced by ${ }^{t} K$, and $\sum \widetilde{p}(x)=1$, but we shall follow [6] in favoring densities with respect to $\widetilde{\pi}$, i.e. densities $\widetilde{\rho}$ verifying $\sum \widetilde{\rho}(x) \widetilde{\pi}(x)=1$. It has been established in [6] that (1), for an appropriate metric $\widetilde{W}_{2}$ which is the discrete counterpart of the standard Wasserstein distance, is a gradient flow of the discrete relative entropy

$$
\widetilde{H}(\widetilde{\rho})=\sum_{x \in V} \widetilde{\rho}(x) \log (\widetilde{\rho}(x)) \widetilde{\pi}(x)
$$




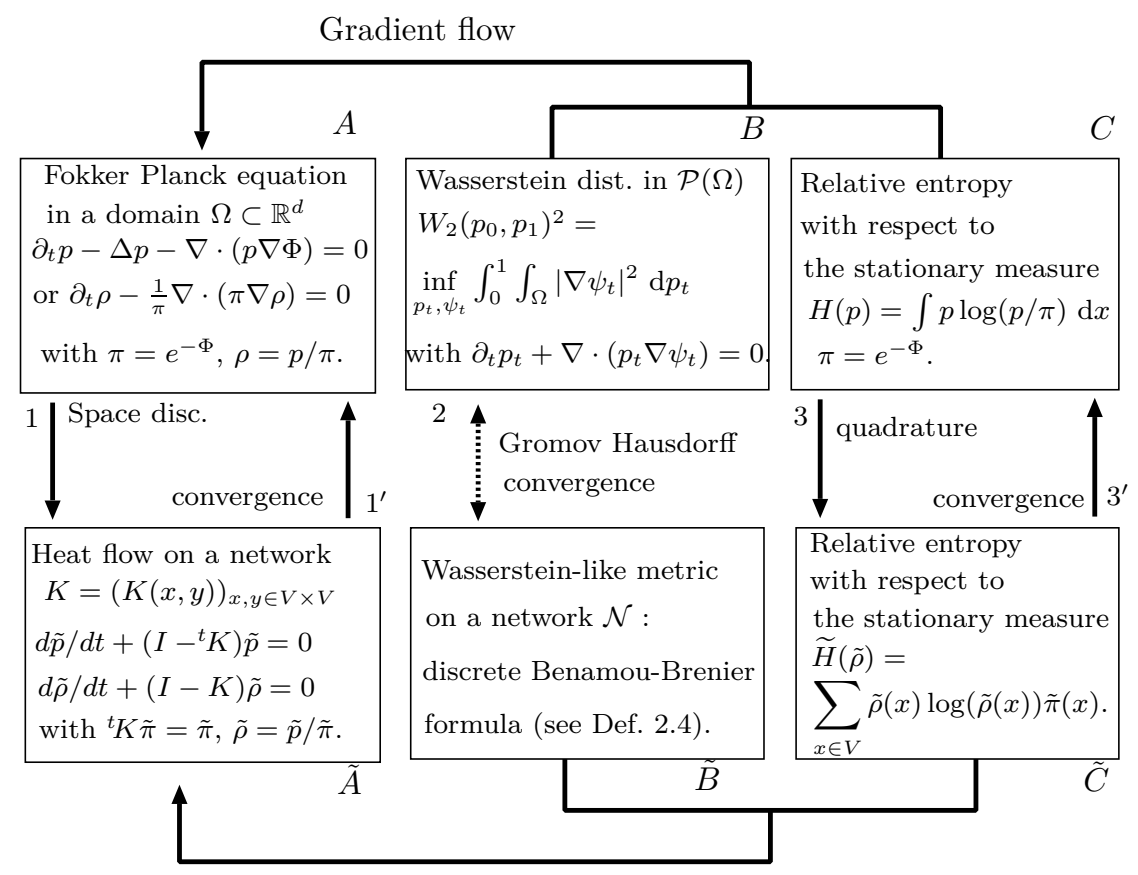

Gradient flow

Fig. 1. Continuous setting versus discrete setting

with respect to the Wasserstein-like metric $\widetilde{W}_{2}$ (see Section 2 for detailed definitions). This discrete setting is also schematized in Fig. 1 (blocks $\tilde{A}-\tilde{B}-\tilde{C}$, on the bottom).

Although it was not the original purpose in [6], a connection can be made between the two settings by means of discretization strategies. As detailed in the next section, an euclidean domain $\Omega$ can be partitionned into cells (e.g. Voronoï cells associated to a collection of points in the domain, see Fig. 2). Now consider the network associated to those cells (one may consider that the vertices are the centroids of the cells). To any measure $\mu$ on $\Omega$ one can associate a discrete measure that is, for each vertex associated to cell $K$, the measure $\mu(K)$. As detailed in [11], a link can be made between the Wasserstein distance on the euclidean domain and the discrete Wasserstein distance on the network, at least in the case of a regular decomposition (cartesian grid). This link will be described more precisely in the next section, it is indicated by the arrow 2 in Fig. 1 that relates blocks $B$ and $\tilde{B}$. Besides, integrating any function of the density at the continuous level has a discrete counterpart (we are especially interested in 
entropy-like functionals), it consists in summing up the corresponding values for the discrete densities built as described above. This approach can be seen as a quadrature formula to compute the approximation of an integral, for which convergence properties can be expected as the cell decomposition is refined. It is indicated by the arrow 3 in Fig. 1 that relates blocks $C$ and $\tilde{C}$.

The core of the present article is an attempt toward closing the diagram by expliciting the link between blocks $A$ and $\tilde{A}$ (arrow 1). More precisely, we aim at showing that, in the context of Finite Volume methods, some space discretization strategies of the FP equation lead to Ordinary Differential Equation that are consistent with the gradient flow structure on the underlying network. Note that this interpretation of Finite Volume discretization schemes as gradient flows has already been addressed in two recent papers. In [2], the authors use this gradient flow structure to characterize the long time behavior of discrete solutions to a fourth order equation. In [1], a finite volume scheme is studied in the discrete Wasserstein setting, and a new type of convergence proof is proposed in this context. In both cited papers, the space discretization is regular (i.e. 1-dimensional for the second one, and d-dimensional with a cartesian grid for the first one). We aim here at showing that an extension to non regular space-discretization is not out of reach. In particular, we show that Finite Volume discretization strategies for very general meshes lead to problems that can be interpreted as gradient flows for a discrete Wasserstein-like metric, with a functional that can be seen as an approximation of its continuous counterpart. Let us make it clear, though, that no discrete-to-continuous convergence result is known for the Wasserstein distance for non regular meshes.

The outline of the paper is as follows. In Section 2 we recall the main obtained result on the FP equation and its gradient flow formulation, we define the Wasserstein-like distance of Maas and state his first result in terms of gradient flows using this distance. Then we describe the Gromov-Hausdorff convergence in the special case of the $d$-dimensional torus and show the convergence of the discrete relative entropy $\widetilde{H}$ to its continuous counterpart $H$. Section 3 proposes a Finite Volume discretization of the FP equation in space and the analysis of the Markov chain deduced from this discretization and seen as an ODE in time. We show that this ODE is the gradient flow of the discrete relative entropy $\widetilde{H}$ and we finalize the paper with some conclusives remarks and perspectives. 


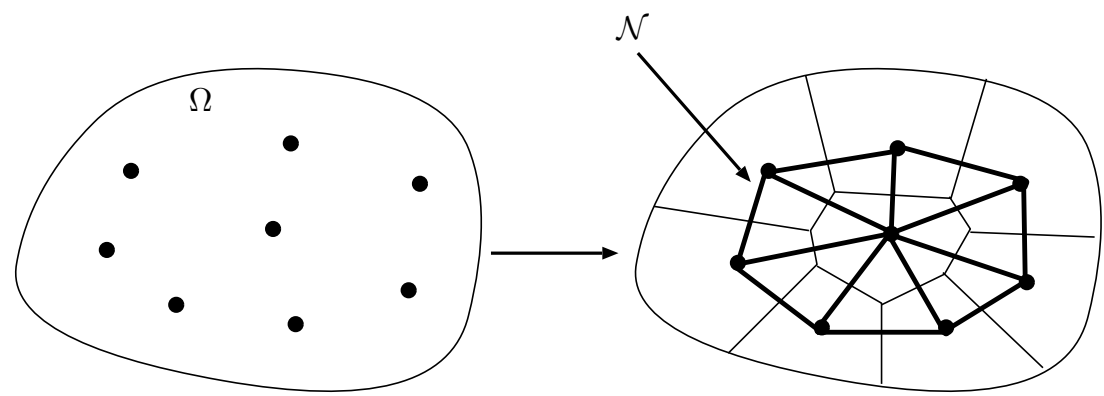

Fig. 2. From the Euclidean domain to the associated network

\section{Preliminaries}

We describe in this section with some details the constitutives blocks of the diagram presented in Fig. 1.

Blocks A-B-C: Fokker Planck equation as a gradient flow, continuous setting

Let us first recall some basic facts on the Wassertein space of measures and gradient flows therein (we refer to $[8,5,4]$ for a detailed presentation of these considerations). Let $\Omega$ be a bounded domain. For any two measures $p_{0}$ and $p_{1}$ in $\mathcal{P}(\Omega)$, the (quadratic) Wasserstein distance between them is defined by

$$
W_{2}\left(p_{0}, p_{1}\right)^{2}=\inf _{\gamma \in \Pi} \int_{\Omega \times \Omega}\left|r^{\prime}-r\right|^{2} \mathrm{~d} \gamma\left(r, r^{\prime}\right),
$$

where $\Pi$ is the subset of $\mathcal{P}(\Omega \times \Omega)$ for all those $\gamma$ with marginals $p_{0}$ and $p_{1}$, respectively, i.e.

$$
\int_{\Omega \times \Omega} \varphi(r) \mathrm{d} \gamma\left(r, r^{\prime}\right)=\int_{\Omega} \varphi(r) \mathrm{d} p_{0}(r), \int_{\Omega \times \Omega} \psi\left(r^{\prime}\right) \mathrm{d} \gamma\left(r, r^{\prime}\right)=\int_{\Omega} \psi\left(r^{\prime}\right) \mathrm{d} p_{0}\left(r^{\prime}\right)
$$

for any continuous functions $\varphi$ and $\psi$.

An alternative formulation has been proposed by Benamou-Brenier [9], it consists in writing the squared Wasserstein distance as follows (we consider here a convex domain):

$$
W_{2}\left(p_{0}, p_{1}\right)^{2}=\inf _{p_{t}, \psi_{t}}\left\{\int_{0}^{1} \int_{\Omega}\left|\nabla \psi_{t}(r)\right|^{2} p_{t}(r) \mathrm{d} x \mathrm{~d} t\right\},
$$


where the infimum runs over curves $\left(p_{t}\right)_{t \in[0,1]}$ in $\mathcal{P}(\Omega)$ that join $p_{0}$ and $p_{1}$ in the following way (transport of $p_{t}$ by $\nabla \psi_{t}$ ):

$$
\partial_{t} p_{t}+\nabla \cdot\left(p_{t} \nabla \psi_{t}\right)=0
$$

Now we aim at defining a notion of gradient for a functional $H$ that is consistent with the Wasserstein framework. The more appropriate notion is that of Fréchet subdifferential in a Wasserstein sense, that can be defined for a wide class of functionals, with very weak smoothness assumptions (see e.g. [4]). Since this notion does not have any natural counterpart at the discrete level, we shall focus here on a more restrictive definition of the gradient:

Definition 2.1. Let $H: \mathcal{P}(\Omega) \rightarrow \mathbb{R}$ be a functional. We shall say that $H$ admits a gradient $w \in L_{p}^{2}$ at $p \in \mathcal{P}(\Omega)$, and then write

$$
\operatorname{grad} H(p)=w,
$$

if, for every measure path $t \rightarrow p_{t}$ defined in a neighborhood of 0 and satisfying:

$$
\frac{\partial p_{t}}{\partial t}+\nabla \cdot\left(p_{t} v_{t}\right)=0, p_{0}=p,
$$

where $v_{t}$ is a $L^{2}$ vector field, it holds that

$$
\left.\frac{d}{d t} H\left(p_{t}\right)\right|_{t=0}=\lim _{t \rightarrow 0} \frac{H\left(p_{t}\right)-H\left(p_{0}\right)}{t}=\int_{\Omega} v_{0} \cdot w d p .
$$

We may now define the notion of gradient flow in this setting:

Definition 2.2. The probability measure path $t \mapsto p_{t}$ is said to be a gradient flow for a functional $H$ if $p_{t}$ verifies (in the distributional sense)

$$
\partial_{t} p_{t}+\nabla \cdot\left(p_{t} u_{t}\right)=0, u_{t}=-\operatorname{grad} H\left(p_{t}\right) \text { for a.e. } t,
$$

where the gradient is defined according to Def. 2.1.

Let us consider the case where $H$ reads

$$
H(p)=\int_{\Omega} f(r) \mathrm{d} p(r)+\int_{\Omega} g(p(r)) \mathrm{d} r,
$$

where $f$ and $g$ are regular functions. Then the transport velocity $u_{t}$ can be identified as

$$
u_{t}=-\nabla f-\nabla\left(g^{\prime}\left(p_{t}\right)\right)
$$


Now consider the Fokker-Planck equation on a domain $\Omega$ :

$$
\left\{\begin{array}{l}
\frac{\partial p}{\partial t}-\Delta p-\nabla \cdot(p \nabla \Phi)=0, \text { in } \Omega \\
\frac{\partial p}{\partial n}-p \frac{\partial \Phi}{\partial n}=0, \text { on } \partial \Omega .
\end{array}\right.
$$

and the relative entropy functional:

$$
\begin{gathered}
H(p)=\int_{\Omega} p(r) \log \left(\frac{p(r)}{\pi(r)}\right) \mathrm{d} r \\
=-\int_{\Omega} \log (\pi(r)) \mathrm{d} p(r)+\int_{\Omega} p(r) \log (p(r)) \mathrm{d} r .
\end{gathered}
$$

We obtain from (5)

$$
u_{t}=-\nabla f-\nabla\left(g^{\prime}\left(p_{t}\right)\right)=\frac{\nabla \pi}{\pi}-\nabla\left(1+\log \left(p_{t}\right)\right)=-\nabla \Phi-\frac{\nabla p_{t}}{p_{t}},
$$

which identifies the FP equation (6) as a gradient flow in the Wasserstein sense for the relative entropy functional $(7)$. We refer again to $[4,5]$ for a thorough presentation of these facts.

\section{Blocks $\tilde{A}-\tilde{B}-\tilde{C}$ : Discrete setting}

Let $V$ be a finite set.

Definition 2.3. We say that $(K(x, y))_{x, y \in V}$ is an irreducible and reversible Markov kernel on $V \times V$ if $K$ satisfies:

1. $K(x, y) \geq 0 \quad \forall x, y \in V, \sum_{y \in V} K(x, y)=1 \quad \forall x \in V$.

2. The irreducibility condition: $K(x, y) \geq 0 \quad \forall x, y \in V, \quad \sum_{y \in V} K(x, y)=$ $1 \forall x \in V$.

3. The reversibility condition: $\tilde{\pi}(x) K(x, y)=K(y, x) \widetilde{\pi}(y) \quad \forall x, y \in V$,

Let $(K(x, y))_{x, y \in V}$ be as in the definition. We denote by $\tilde{\pi}$ the unique stationary measure of $K$, such that

$$
\left.\tilde{\pi}(x)=\sum_{y \in V} K(y, x) \widetilde{\pi}(y) \quad \text { (i.e. } \tilde{\pi}={ }^{t} K \widetilde{\pi}\right), \tilde{\pi}(x)>0 \quad \forall x \in V, \sum_{x \in V} \tilde{\pi}(x)=1 .
$$

We define the associated set of probability densities on $V$ by

$$
\mathscr{D}(V)=\left\{\widetilde{\rho} \in\left(\mathbb{R}_{+}\right)^{V}, \sum_{x \in V} \widetilde{\rho}(x) \widetilde{\pi}(x)=1\right\} .
$$

Following [6], we define the discrete gradient, the discrete divergence and the two scalar products with respect to a fixed $\widetilde{\rho} \in \mathscr{D}(V)$ and $\tilde{\pi}$ resp., as follows: 
- Discrete gradient: For a function $\widetilde{\psi}: V \rightarrow \mathbb{R}$, we define its discrete gradient $\widetilde{\nabla} \widetilde{\psi}: V \times V \rightarrow \mathbb{R}$ by

$$
\widetilde{\nabla} \widetilde{\psi}(x, y)=\widetilde{\psi}(y)-\widetilde{\psi}(x) \quad \forall x, y \in V \times V .
$$

- Discrete divergence: For a discrete field $\widetilde{u}: V \times V \rightarrow \mathbb{R}$, we define its discrete divergence $\widetilde{\nabla} \cdot \widetilde{u}: V \rightarrow \mathbb{R}$ by

$$
(\widetilde{\nabla} \cdot \widetilde{u})(x)=\frac{1}{2} \sum_{y \in V}(\widetilde{u}(x, y)-\widetilde{u}(y, x)) K(x, y) \quad \forall x \in V .
$$

Note that for an anti-symmetric field u, i.e. $\widetilde{u}(x, y)=-\widetilde{u}(y, x)$, the discrete divergence reads:

$$
(\widetilde{\nabla} \cdot \widetilde{u})(x)=\sum_{y \in V}(\widetilde{u}(x, y)) K(x, y) \quad \forall x \in V .
$$

- Scalar product with respect to $\tilde{\pi}:$ For $\widetilde{\psi}, \widetilde{\phi}: V \rightarrow \mathbb{R}$, we define their scalar product with respect to $\tilde{\pi}$ by

$$
\langle\langle\widetilde{\psi}, \widetilde{\phi}\rangle\rangle_{\widetilde{\pi}}=\sum_{x \in V} \widetilde{\psi}(x) \widetilde{\phi}(x) \widetilde{\pi}(x) .
$$

- Scalar product with respect to $\widetilde{\rho}$ : For $\widetilde{u}, \widetilde{v}: V \times V \rightarrow \mathbb{R}$, we define their scalar product with respect to $\widetilde{\rho}$ by

$$
\langle\widetilde{u}, \widetilde{v}\rangle_{\widetilde{\rho}}=\frac{1}{2} \sum_{x, y \in V} \widetilde{u}(x, y) \widetilde{v}(x, y) K(x, y) \theta(\widetilde{\rho}(x), \widetilde{\rho}(y)) \widetilde{\pi}(x),
$$

where $\theta(\cdot, \cdot)$ is defined by $(9)$, and we denote by $\|\widetilde{u}\|_{\sim}$ the associated norm:

$$
\|\widetilde{u}\|_{\widetilde{\rho}}=\sqrt{\langle\widetilde{u}, \widetilde{u}\rangle_{\tilde{\rho}}}
$$

Note that the latter is a discrete counterpart of the norm of a velocity field in $L_{p}^{2}$, with $p=\rho \pi$.

We denote by $\langle., .\rangle_{\mathbf{1}}$ the scalar product with respect to the density $\widetilde{\rho}=\mathbf{1}$ defined by: $\widetilde{\rho}(x)=1, \forall x \in V$. We can easily check that the integration by parts formula holds in the following sense:

$$
\langle\widetilde{\nabla} \widetilde{\psi}, \widetilde{u}\rangle_{\mathbf{1}}=-\langle\langle\widetilde{\psi}, \widetilde{\nabla} \cdot \widetilde{u}\rangle\rangle_{\widetilde{\pi}}
$$

The definition of the discrete transportation metric is inspired by the Benamou-Brenier formulation, it translates Eq. (4) at the discrete level. It is defined as (see $[6,10,7])$ : 
Definition 2.4. For $\widetilde{\rho}_{0}, \widetilde{\rho}_{1} \in \mathscr{D}(V)$ we set:

$$
\widetilde{W}_{2}\left(\widetilde{\rho}_{0}, \widetilde{\rho}_{1}\right)^{2}=\inf _{\widetilde{\rho}_{t}, \widetilde{\psi}_{t}}\left\{\frac{1}{2} \int_{0}^{1} \sum_{x, y \in V}\left(\widetilde{\psi}_{t}(y)-\widetilde{\psi}_{t}(x)\right)^{2} K(x, y) \theta\left(\widetilde{\rho}_{t}(x), \widetilde{\rho}_{t}(y)\right) \widetilde{\pi}(x) \mathrm{d} t\right\}
$$

where the infimum runs over all piecewise $C^{1}$ curves $\widetilde{\rho}_{t}:[0,1] \rightarrow \mathscr{D}(V)$ and all piecewise continuous $\widetilde{\psi}_{t}:[0,1] \rightarrow \mathbb{R}^{V}$ satisfying:

$$
\frac{\mathrm{d} \widetilde{\rho}_{t}}{\mathrm{~d} t}(x)+\sum_{y \in V}\left(\widetilde{\psi}_{t}(y)-\widetilde{\psi}_{t}(x)\right) K(x, y) \theta\left(\widetilde{\rho}_{t}(x), \widetilde{\rho}_{t}(y)\right)=0 \quad \forall x \in V
$$

where:

$$
\theta(\alpha, \beta)=\int_{0}^{1} \alpha^{1-t} \beta^{t} d t= \begin{cases}\frac{\beta-\alpha}{\log (\beta)-\log (\alpha)}, & \text { if } \alpha \neq \beta \\ \alpha, & \text { if } \alpha=\beta\end{cases}
$$

is the logarithmic mean of $\alpha$ and $\beta$.

Using the definitions of the discrete gradient and the discrete divergence, $\widetilde{W}_{2}$ can also be formulated as follows (see [6], Lemma 3.5):

$$
\widetilde{W}_{2}\left(\widetilde{\rho}_{0}, \widetilde{\rho}_{1}\right)^{2}=\inf _{\widetilde{\rho}_{t}, \widetilde{\psi}_{t}}\left\{\int_{0}^{1}\left\|\widetilde{\nabla} \widetilde{\psi}_{t}\right\|_{\tilde{\rho}_{t}}^{2} \mathrm{~d} t\right\}
$$

where the infimum runs over all piecewise $C^{1}$ curves $\left(\widetilde{\rho}_{t}\right)_{t \in[0,1]}$ joining $\widetilde{\rho}_{0}$ and $\widetilde{\rho}_{1}$ in $\mathscr{D}(V)$ according to

$$
\frac{\mathrm{d} \widetilde{\rho}_{t}}{\mathrm{~d} t}(x)+\widetilde{\nabla} \cdot\left(\Theta\left(\widetilde{\rho}_{t}\right) \bullet \widetilde{\nabla} \widetilde{\psi}\right)(x)=0, \forall x \in V
$$

where $\Theta\left(\widetilde{\rho}_{t}\right): V \times V \rightarrow \mathbb{R}$ is defined by: $\Theta\left(\widetilde{\rho}_{t}\right)(x, y)=\theta\left(\widetilde{\rho}_{t}(x), \widetilde{\rho}_{t}(y)\right)$ and • denotes the entrywise product of two matrices.

The Wasserstein gradient of a functional may now be defined following [6], Prop. 4.2 .

Definition 2.5. Let $\widetilde{H}: \mathscr{D}(V) \rightarrow \mathbb{R}$ be a functional. We shall say that $\widetilde{H}$ admits a gradient $\widetilde{w} \in \mathbb{R}^{V \times V}$ at $\widetilde{\rho} \in \mathscr{D}(V)$, and then write

$$
\widetilde{\operatorname{grad}} \widetilde{H}(\widetilde{\rho})=\widetilde{w},
$$

if, for any measure path $t \rightarrow \widetilde{\rho}_{t}$ on $\mathscr{D}(V)$ defined in a neighborhood of 0 , with

$$
\frac{\mathrm{d} \widetilde{\rho}_{t}}{\mathrm{~d} t}+\widetilde{\nabla} \cdot\left(\Theta\left(\widetilde{\rho}_{t}\right) \bullet \widetilde{v}_{t}\right)=0, \quad \widetilde{\rho}_{0}=\widetilde{\rho},
$$

it holds that

$$
\left.\frac{\mathrm{d}}{\mathrm{d} t} \widetilde{H}\left(\widetilde{\rho}_{t}\right)\right|_{t=0}=\left\langle\widetilde{w}, \widetilde{v}_{0}\right\rangle_{\tilde{\rho}}
$$


After computing the gradient of a functional $\widetilde{H}$, we can write its gradient flow equation in $\mathscr{D}(V)$.

Definition 2.6. Let $\widetilde{H}: \mathscr{D}(V) \rightarrow \mathbb{R}$ be a functional and $\widetilde{\operatorname{grad}} \widetilde{H}$ be its gradient according to Def (2.5). We define the discrete gradient flow equation of $\widetilde{H}$ by:

$$
\frac{\mathrm{d} \widetilde{\rho}}{\mathrm{d} t}(x)-\widetilde{\nabla} \cdot(\Theta(\widetilde{\rho}) \bullet \widetilde{\operatorname{grad}} \widetilde{H}(\widetilde{\rho}))(x)=0 \quad \forall x \in V .
$$

Like in the continuous setting, the gradient (in the previous sense) of a certain class of functionals can be computed explicitly.

Proposition 2.7. Let $\widetilde{H}$ be a generalized entropy functional :

$$
\widetilde{H}: \widetilde{\rho} \in \mathscr{D}(V) \longmapsto \widetilde{H}(\widetilde{\rho})=\sum_{x \in V} f(x) \widetilde{\rho}(x) \widetilde{\pi}(x)+\sum_{x \in V} g(\widetilde{\rho}(x)) \widetilde{\pi}(x) \in \mathbb{R}
$$

where $f, g$ are differentiable function, $f, r:(0,1) \rightarrow \mathbb{R}$. Then

$$
\left.\widetilde{\operatorname{grad}} \widetilde{H}(\widetilde{\rho})=\widetilde{\nabla} f+\widetilde{\nabla} g^{\prime} \circ \widetilde{\rho}\right) \text {. }
$$

Proof. It is a straightforward application of the definitions above

$$
\begin{gathered}
\left.\frac{\mathrm{d}}{\mathrm{d} t} \widetilde{H}\left(\widetilde{\rho}_{t}\right)\right|_{t=0}=\left.\sum_{x \in V}\left(f(x)+g^{\prime}\left(\widetilde{\rho}_{t}(x)\right)\right) \frac{\mathrm{d} \widetilde{\rho}_{t}}{\mathrm{~d} t}(x) \widetilde{\pi}(x)\right|_{t=0} \\
=-\left.\sum_{x \in V}\left(f(x)+g^{\prime}\left(\widetilde{\rho}_{t}(x)\right)\right) \widetilde{\nabla} \cdot\left(\Theta\left(\widetilde{\rho}_{t}\right) \bullet \widetilde{v}_{t}\right)(x) \widetilde{\pi}(x)\right|_{t=0} \\
=-\left\langle\left\langle f+g^{\prime} \circ \widetilde{\rho}, \widetilde{\nabla} \cdot\left(\Theta(\widetilde{\rho}) \bullet \tilde{v}_{0}\right)\right\rangle\right\rangle_{\widetilde{\pi}}=\left\langle\widetilde{\nabla}\left(f+g^{\prime} \circ \widetilde{\rho}\right), \Theta(\widetilde{\rho}) \bullet \tilde{v}_{0}\right\rangle_{1} \\
=\left\langle\widetilde{\nabla} f+\widetilde{\nabla} g^{\prime} \circ \widetilde{\rho}, \tilde{v}_{0}\right\rangle_{\widetilde{\rho}}
\end{gathered}
$$

which concludes the proof.

\section{Heat flow equation as gradient flow of the discrete entropy}

Note that the heat flow equation

$$
\frac{\mathrm{d} \widetilde{\rho}}{\mathrm{d} t}+(I-K) \widetilde{\rho}=0
$$

where $K=(K(x, y))_{x, y}$ is the Markov matrix, can also be written

$$
\frac{\mathrm{d} \widetilde{\rho}}{\mathrm{d} t}(x)-\nabla \cdot(\nabla \widetilde{\rho})(x)=0 \quad \forall x \in V .
$$

We may now identify the heat flow equation with the gradient flow for the relative entropy. 
Theorem 2.8. The gradient flow in $\mathscr{D}(V)$ (according to Def (2.6)) of the discrete relative entropy

$$
\widetilde{H}(\widetilde{\rho})=\sum_{x \in V} \widetilde{\rho}(x) \log (\widetilde{\rho}(x)) \widetilde{\pi}(x)
$$

is the heat flow equation (11).

Proof. For a detailed proof, we refer the reader to [6], Theorem 1.2. From Proposition 2.7 , we have that

$$
\widetilde{\operatorname{grad}} \widetilde{H}(\widetilde{\rho})=\widetilde{\nabla}(1+\log (\widetilde{\rho}))=\widetilde{\nabla}(\log (\widetilde{\rho}))
$$

for $g(\widetilde{\rho})=\widetilde{\rho} \log \widetilde{\rho}$ and $f=0$, and the discrete gradient flow equation of $\widetilde{H}$ is:

$$
\frac{\mathrm{d} \widetilde{\rho}}{\mathrm{d} t}(x)-\widetilde{\nabla} \cdot(\Theta(\widetilde{\rho}) \bullet \widetilde{\nabla}(\log (\widetilde{\rho})))(x)=0 \quad \forall x \in V .
$$

Link $B-\tilde{B}$ (arrow 2): Link between continuous and discrete Wasserstein metrics

A first result of convergence of the discrete transportation metric was proven in [11] (Theorem 3.15).

We consider the space $\mathcal{P}\left(\mathbb{T}^{d}\right)$ of all the probability measures on the $d$ dimensional torus $\mathbb{T}^{d}=\mathbb{R}^{d} / \mathbb{Z}^{d}$ endowed with the $L^{2}$-Wasserstein metric and the $d$-dimensional periodic lattice $\mathbb{T}_{n}^{d}=(\mathbb{Z} / n \mathbb{Z})^{d}$ and endow the space of probability densities $\mathscr{D}\left(\mathbb{T}_{n}^{d}\right)$ with the renormalised discrete transportation metric $\widetilde{W}_{2, n}=\widetilde{W}_{2} / n \sqrt{2 d}$ where the Markov kernel $K$ is the one of a simple random walk (uniform transition probabilities) and whose stationary measure $\widetilde{\delta}$ is the uniform measure on $\mathbb{T}_{n}^{d}$.

In this special case of $\widetilde{\delta}$, we can identify probability measures on $\mathbb{T}_{n}^{d}$ with their probability densities with respect to $\widetilde{\delta}$. So we consider that $\mathscr{P}\left(\mathbb{T}_{n}^{d}\right) \equiv$ $\mathscr{D}\left(\mathbb{T}_{n}^{d}\right)$.

The convergence result is established in the sense of Gromov-Hausdorff that is defined by:

Definition 2.9. A sequence of compact metric spaces $\left(\mathcal{X}_{n}, d_{n}\right)$ is said to converge in the sense of Gromov-Hausdorff to a compact metric space $(\mathcal{X}, d)$, if there exists a sequence of maps $f_{n}: \mathcal{X} \rightarrow \mathcal{X}_{n}$ which are:

- $\epsilon_{n}$-isometric, i.e., for all $x, y \in \mathcal{X}$,

$$
\left|d_{n}\left(f_{n}(x), f_{n}(y)\right)-d(x, y)\right| \leq \epsilon_{n}
$$


- $\epsilon_{n}$-surjective, i.e., for all $x_{n} \in \mathcal{X}_{n}$ there exists $x \in \mathcal{X}$ with

$$
d\left(f_{n}(x), x_{n}\right) \leq \epsilon_{n}
$$

for some sequence $\epsilon_{n} \rightarrow 0$.

Now we are ready to state the convergence theorem of the discrete metrics $\widetilde{W}_{2, n}$ :

Theorem 2.10. The metric spaces $\left(\mathscr{P}\left(\mathbb{T}_{n}^{d}\right), \widetilde{W}_{2, n}\right)$ converge to $\left(\mathcal{P}\left(\mathbb{T}^{d}\right), W_{2}\right)$ in the sense of Gromov-Hausdorff as $n \rightarrow \infty$.

Remark 2.11. An informal convergence result can be done for a general stationary measure by discretizing the continuous FP equation with the scheme described in Section 3 and writing the corresponding discrete distance which looks almost like a discretization of the continuous Wasserstein distance.

\section{Link $\tilde{C}-\tilde{C}$ (arrow 3): Quadrature for the entropy functionnal}

In order to strenghen the relation between the discrete setting and the continuous one, we are going to show the convergence of the discrete relative entropy to its continuous counterpart $(\tilde{C} \longrightarrow C$ in the Diagram of Fig. 1). We consider a collection of $n$ points $V$ in $\Omega$, and construct a partition $\left(K_{x}\right)_{x}$ of the domain which is relative to $V$, i.e. each cell $K_{x}$ of the partition contains one point of $V$ (which is $x$ ) and:

$$
\bar{\Omega}=\bigcup_{x \in V} \bar{K}_{x}, K_{x} \cap K_{y}=\emptyset \quad \forall x, y \in V .
$$

Let $h$ be the diameter of the partition $K_{x}$, i.e. $h=\max _{x \in V} \operatorname{diam}\left(K_{x}\right)$. For any probability measure $p$ in $\mathcal{P}(\Omega)$, we define its discrete counterpart by:

$$
\widetilde{p}(x)=\int_{K_{x}} p(r) \mathrm{d} r, \forall x \in V, \widetilde{p} \in \mathscr{P}(V)
$$

and then we define its discrete density by:

$$
\widetilde{\rho}(x)=\frac{\widetilde{p}(x)}{\widetilde{\pi}(x)}, \forall x \in V, \widetilde{\rho} \in \mathscr{D}(V) .
$$

Proposition 2.12. Let $p, \pi$ be two $C^{1}(\bar{\Omega})$ densities with respect to the Lebesgue measure, bounded from below and above, i.e. $\exists m, M>0$ such that $0<m \leq$ $p, \pi \leq M$, and $\widetilde{p}_{h}, \widetilde{\pi}_{h}$ be their discrete counterpart defined according to (12). 
We denote by $\widetilde{\rho}_{h}$ the discrete density of $\widetilde{p}_{h}$ with respect to $\tilde{\pi}_{h}$. Then, the discrete relative entropy:

$$
\widetilde{H}_{h}\left(\widetilde{\rho}_{h}\right)=\sum_{x \in V_{h}} \widetilde{\rho}_{h}(x) \log \left(\widetilde{\rho}_{h}(x)\right) \widetilde{\pi}_{h}(x)
$$

converges to the continuous relative entropy:

$$
H(p)=\int_{\Omega} p(r) \log \left(\frac{p(r)}{\pi(r)}\right) \mathrm{d} r
$$

when $h \rightarrow 0$, at the first order in $h$.

Proof. We substract the continuous and the discrete quantities:

$$
\begin{aligned}
\left|H(p)-\widetilde{H}\left(\widetilde{\rho}_{h}\right)\right|= & \left|\sum_{x \in V}\left(\int_{K_{x}} p(r) \log \left(\frac{p(r)}{\pi(r)}\right) \mathrm{d} r-\widetilde{p}(x) \log \left(\frac{\widetilde{p}(x)}{\widetilde{\pi}(x)}\right)\right)\right| \\
= & \left|\sum_{x \in V_{K_{x}}} \int_{p(r)}\left(\log \left(\frac{p(r)}{\pi(r)}\right)-\log \left(\frac{\tilde{p}(x)}{\widetilde{\pi}(x)}\right)\right) \mathrm{d} r\right| \\
& \leq \sum_{x \in V_{K_{x}}} \int_{\mid} p(r) C\left|\frac{p(r)}{\pi(r)}-\frac{\widetilde{p}(x)}{\widetilde{\pi}(x)}\right| \mathrm{d} r
\end{aligned}
$$

where $C$ is a Lipchitz constant for $\log$ on $\left[\frac{m}{M}, \frac{M}{m}\right]$. Then, by straightforward computations using the boundedness from below and above of the measureswe can bound the substraction by $C^{\prime} \times h$ where $C^{\prime}$ is a constant depending on $m, M$ and $C$.

\section{Discretization of the Fokker-Planck equation}

We re-write the Fokker-Planck system by replacing $\nabla \Phi$ by $-\nabla \pi / \pi$ in the first equation, we get:

$$
\frac{\partial p}{\partial t}-\nabla \cdot\left(\nabla p-p \frac{\nabla \pi}{\pi}\right)=0, \text { or equivalently: } \frac{\partial p}{\partial t}-\nabla \cdot\left(\pi \nabla\left(\frac{p}{\pi}\right)\right)=0
$$

\section{Finite volume discretization}

Let $V$ be a collection of points in $\Omega$, and let $\left(K_{x}\right)_{x}$ be the associated Voronoi tesselation. We denote by $\mathcal{N}$ the dual network of the space discretization (two 


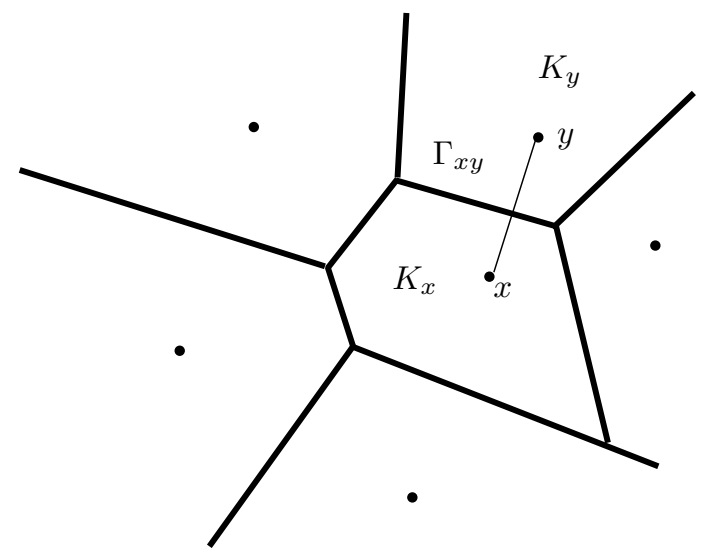

Fig. 3. Voronoi cells with the used notations

vertices are connected whenever the corresponding cells are adjacent). Then we discretize in space the FP equation (in its form (13)) by a Finite Volume scheme (see e.g. [12]). By integrating the equation on $K_{x}$, we get:

$$
\frac{\mathrm{d} \tilde{p}}{\mathrm{~d} t}(x)-\int_{\partial K_{x}} \pi \nabla\left(\frac{p}{\pi}\right) n_{x} \mathrm{~d} \sigma=\frac{\mathrm{d} \widetilde{p}}{\mathrm{~d} t}(x)-\sum_{y \sim x_{\Gamma_{x y}}} \int_{x} \pi\left(\frac{p}{\pi}\right) n_{x y} \mathrm{~d} \sigma=0
$$

where $n_{x}$ is the outward normal to $K_{x}, \Gamma_{x y}=\overline{K_{x}} \cap \overline{K_{y}}, n_{x y}=\left.n_{x}\right|_{\Gamma_{x y}}$ and $y \sim x$ means that $y$ is a neighbor of $x, y \neq x$.

We approximate

$$
\int_{\Gamma_{x y}} \pi \nabla\left(\frac{p}{\pi}\right) n_{x y} \mathrm{~d} \sigma \quad \text { by } \quad \frac{\left|\Gamma_{x y}\right|}{|x-y|} \theta(\widetilde{\pi}(x), \widetilde{\pi}(y))\left(\frac{\widetilde{p}(y)}{\widetilde{\pi}(y)}-\frac{\widetilde{p}(x)}{\widetilde{\pi}(x)}\right)
$$

(this approximation is inspired from [13] and was used in [1] for the 1-dimensional case) and we get the final form of the semi-discretized equation:

$$
\frac{\mathrm{d}}{\mathrm{d} t} \widetilde{p}(x)=\sum_{y \sim x} \frac{\left|\Gamma_{x, y}\right|}{|x-y|} \theta(\widetilde{\pi}(x), \widetilde{\pi}(y))\left(\frac{\widetilde{p}(y)}{\widetilde{\pi}(y)}-\frac{\widetilde{p}(x)}{\widetilde{\pi}(x)}\right)
$$

Or equivalently:

$$
\begin{gathered}
\frac{\mathrm{d}}{\mathrm{d} t} \widetilde{p}(x)=\sum_{y \sim x}\left(\frac{\left|\Gamma_{x y}\right|}{|x-y|} \theta(\widetilde{\pi}(x), \widetilde{\pi}(y)) \frac{\widetilde{p}(y)}{\widetilde{\pi}(y)}\right) \\
-\left(\sum_{y \sim x} \frac{\left|\Gamma_{x y}\right|}{|x-y|} \theta(\widetilde{\pi}(x), \widetilde{\pi}(y))\right) \frac{\widetilde{p}(x)}{\widetilde{\pi}(x)}
\end{gathered}
$$


which can be seen as an evolution equation on the network $\mathcal{N}$.

\section{Semi-discretized equation written with probability densities:}

An equivalent semi-discretized equation of (14) is written with the probability densities $\widetilde{\rho}$ with respect to $\tilde{\pi}$, i.e. $\widetilde{\rho}(x)=\widetilde{p}(x) \widetilde{\pi}(x)$ :

$$
\begin{gathered}
\frac{\mathrm{d}}{\mathrm{d} t} \widetilde{\rho}(x)=\sum_{y \sim x}\left(\frac{\left|\Gamma_{x y}\right|}{|x-y| \widetilde{\pi}(x)} \theta(\widetilde{\pi}(x), \widetilde{\pi}(y)) \widetilde{\rho}(y)\right) \\
-\left(\sum_{y \sim x} \frac{\left|\Gamma_{x y}\right|}{|x-y| \widetilde{\pi}(x)} \theta(\widetilde{\pi}(x), \widetilde{\pi}(y))\right) \widetilde{\rho}(x) .
\end{gathered}
$$

Now, equation (15) can be written

$$
\frac{\mathrm{d}}{\mathrm{d} t} \widetilde{\rho}=Q \widetilde{\rho}
$$

with

$$
Q(x, y)= \begin{cases}\frac{\left|\Gamma_{x y}\right| \theta(\widetilde{\pi}(x), \tilde{\pi}(y))}{|x-y| \widetilde{\pi}(x)}, & \text { if } x \sim y \\ -\sum_{y \sim x} \frac{\left|\Gamma_{x y}\right| \theta(\widetilde{\pi}(x), \tilde{\pi}(y))}{|x-y| \widetilde{\pi}(x)}, & \text { if } x=y \\ 0 & \text { otherwise }\end{cases}
$$

on the network $\mathcal{N}$.

Equation (16) is not exactly of the heat flow type (11), since $Q$ is not of the form $K-I$, where $K$ would be a stochastic matrix. Yet, as pointed out in [14], a connection can be made between the two settings: For a matrix $Q$ as above, we set

$$
q_{x}=\sum_{y \sim x} Q(x, y) \quad \text { and } \quad q_{\max }=\max _{x} q_{x} .
$$

We then define the matrix $K$ as follows:

$$
K(x, y)= \begin{cases}\frac{Q(x, y)}{q_{\max }}, & \text { if } x \neq y \\ \frac{q_{\max }-q_{x}}{q_{\max }}, & \text { if } x=y\end{cases}
$$

Proposition 3.1. The matrix $K$ resulting from the space discretization of the FP equation (13) as described above is an irreducible and reversible Markov Kernel that admits $\tilde{\pi}$ as stationary measure.

Proof. The matrix $K$ has the following properties: 
(i) $K(x, y)=Q(x, y) / q_{\max } \geq 0$ for $x \neq y, K(x, x)=\left(q_{\max }-q_{x}\right) / q_{\max } \geq 0$, and

$$
\begin{gathered}
\sum_{y \in V} K(x, y)=\sum_{y \sim x} \frac{Q(x, y)}{q_{\max }}+\frac{q_{\max }-q_{x}}{q_{\max }} \\
=\sum_{y \sim x} \frac{Q(x, y)}{q_{\max }}+1-\sum_{y \sim x} \frac{Q(x, y)}{q_{\max }}=1 .
\end{gathered}
$$

(ii) $K(x, y) \neq 0$ for $x \sim y$ and the network is strongly connected, we deduce that $K$ is irreducible and then has a unique stationary measure.

(iii) $\tilde{\pi}$ satisfies the detailed balance equation for all $x, y \in V$ :

$$
\begin{aligned}
& \tilde{\pi}(x) K(x, y)=\tilde{\pi}(x) \frac{Q(x, y)}{q_{\max }}=\frac{\tilde{\pi}(x)}{q_{\max }} \frac{\left|\Gamma_{x y}\right| \theta(\tilde{\pi}(x), \tilde{\pi}(y))}{|x-y| \tilde{\pi}(x)} \\
& =\frac{\tilde{\pi}(y)}{q_{\max }} \frac{\left|\Gamma_{x y}\right| \theta(\tilde{\pi}(x), \tilde{\pi}(y))}{|x-y| \tilde{\pi}(y)}=\tilde{\pi}(y) \frac{Q(y, x)}{q_{\max }}=\tilde{\pi}(y) K(y, x)
\end{aligned}
$$

so $K$ is reversible, and we have:

$$
\sum_{y \in V} \tilde{\pi}(y) K(y, x)=\sum_{y \in V} \tilde{\pi}(x) K(x, y)=\tilde{\pi}(x)
$$

which proves that $\tilde{\pi}$ is the unique stationary measure of $K$.

By definition of $K$, we have that

$$
\frac{1}{q_{\max }} Q=(K-I),
$$

so that the solution to (16), that is the space-discretized solution, is the solution to the heat flow equation

$$
\frac{\mathrm{d}}{\mathrm{d} t} \widetilde{\rho}+(I-K) \widetilde{\rho}=0
$$

up to an affine time renormalization.

Now recall that the continuous FP equation is the gradient flow in the Wasserstein sense (see Def(2.1)) for the relative entropy (7). We have the following discrete counterpart of this property for the Finite Volume discretization scheme (16), that is a direct consequence of the previous developments:

Proposition 3.2. The space discretized scheme (16) is a gradient flow for the discrete relative entropy (2), up to an affine time renormalization, with respect to the discrete Wasserstein distance $\widetilde{W}_{2}$ (see Def 2.4). 


\section{Conclusives remarks, perspectives}

We described in this paper how some space discretization Finite Volume schemes, possibly on unstructured meshes, can be proved to be deeply respectfull of the underlying gradient flow structure. Given a PDE that is the Wasserstein gradient flow of some functionnal, the ODE resulting from space discretization can be identified as a gradient flow for a discrete functionnal that is an approximation of the continuous one, in the Wasserstein space of measures defined on the underlying network, the vertices of which are the finite volume cells. This overall consistency with respect to Wasserstein metric, that is expressed by Fig. 1, can be used to improve the numerical analysis of a scheme, e.g. by charaterizing its long-time behaviour (as in [2] in the case of a cartesian mesh). Note that the approach is currently limited to the semi-discretized scheme. Let us add that the considered scheme treats the advection in a diffusive manner, and as such it is intrinsically of the centered type, so that stability issues can be expected. In particular, an Euler Explicit scheme is likely to lead to unconditionnal unstability. Implicit time-stepping may, in the contrary, provide some stability. Note that Implicit Euler time-stepping applied to (16) leads to a problem that is formally very similar to the so-called JKO scheme applied at the discrete level to compute the gradient flow. Implicit schemes are then likely to recover some properties of the JKO one .

Let us finally stress that the diagram of Fig. 1 is not fully realized. Indeed, the arrow 2 between blocks $B$ and $B^{\prime}$, which expresses a link between the Wasserstein distance in a domain, and the discrete Wasserstein distance on the network obtained by space discretization, is not covered by a full theory. The only known convergence results ([11]) concern cartesian grids, in the case without potential. In the presence of a non-constant potential, the framework that has been presented may appear puzzling, because the discrete Wasserstein distance involves the stationnary measure (non uniform in general), which depends on the potential $\Phi$, whereas its continuous counterpart pertains to the flat domain, and therefore does not depend on $\Phi$. This apparent paradox is due to the fact that, at the discrete level, the distance $\widetilde{W}_{2}$ is defined for densities with respect to the stationary measure $\tilde{\pi}$. Comparing both distances would amount to consider two probabilties $p_{0}$ and $p_{1}$, compute their discrete counterparts $\widetilde{p}_{0}$ and $\widetilde{p}_{1}$, together with $\tilde{\pi}$, then $\widetilde{\rho}_{0}=\widetilde{p}_{0} / \widetilde{\pi}, \widetilde{\rho}_{1}=\widetilde{p}_{1} / \widetilde{\pi}$, and finally estimate $\widetilde{W}_{2}\left(\widetilde{\rho}_{0}, \widetilde{\rho}_{1}\right)$, and check that the latter converges to $W_{2}\left(p_{0}, p_{1}\right)$ when the discretization is refined. Although not covered by any theoretical result, and in spite of the fact that $\widetilde{W}_{2}$ "sees" the measure $\pi$, while $W_{2}$ does not, such a property can be expected, because $\pi$ is involved twice in the discretization process : firstly by computation of 
$\widetilde{\rho}$ from $\widetilde{p}$, and then, in a hidden way, through the definition of $\widetilde{W}_{2}$. One can check in very simple situations that both effect tend to compensate each other, i.e. the dependence of $\widetilde{W}_{2}\left(\widetilde{\rho}_{0}, \widetilde{\rho}_{1}\right)$ upon $\pi$ asymptotically vanishes. It can also be seen in the very definition of the distance itself: each time $\widetilde{\pi}(x)$ is involved, it is multiplied by a quantity of the type $\theta(\widetilde{\rho}(x), \widetilde{\rho}(y))$, where $x$ and $y$ are connected. In the context of Finite Volume schemes, when the discretization is refined, $x$ and $y$ get closer, so that this quantity is asymptotically close to $\widetilde{\rho}(x)$, and finally the real dependence is upon $\widetilde{p}(x)$, which does no longer depend on the stationnary measure. As for non cartesian meshes, the analogy that we established advocate for a convergence of the discrete Wasserstein metric toward the continuous one, but it remains to be rigorously proven.

Acknowledgment: The authors would like to thank F. Santambrogio for interesting discussions and suggestions. Both authors are partially supported by ANR Project Isotace (ANR-12-MONU-0013).

\section{References}

[1] K. Disser, M. Liero, "On gradient structures for Markov chains and the passage to Wasserstein gradient flows", Networks and Heterogeneous Media, AIMS, 10(2), Pages 233 - 253, (2015).

[2] Jan Maas, Daniel Matthes, “Long-time behavior of a finite volume discretization for a fourth order diffusion equation", arXiv:1505.03178.

[3] R. Jordan, D. Kinderlehrer, F. Otto, "The variational formulation of the Fokker-Planck equation", SIAM Journal of Mathematical Analysis, 29(1), Pages 1-17 (1998).

[4] L. Ambrosio, N. Gigli, G. Savaré, "Gradient flows-In Metric Spaces and in the Space of Probability Measures", Lectures in Mathematics ETH Zurich, Birkhauser Basel, (2008).

[5] F. Santambrogio, "Optimal transport for applied mathematicians", Progress in Nonlinear Differential Equations and their applications 87, Birkhauser Basel (2015).

[6] J. Maas, "Gradient flows of the relative entropy for finite Markov chains", Journal of Functional Analysis, 261(8), Pages 2250-2292 (2011).

[7] A. Mielke, "A gradient structure for reaction-diffusion systems and for energy-driftdiffusion systems", Non-linearity, 24(4), Pages 1329-1346 (2011).

[8] C. Villani, "Optimal transport, old and new", Grundlehren der Mathematischen Wissenschaften, Springer-Verlag (2013).

[9] J.-D. Benamou and Y. Brenier, "A computational fluid mechanics solution to the Monge-Kantorovich mass transfer problem", Numerische Mathematik, 84, Pages 375393 (2000).

[10] S.-N. Chow, W. Huang, Y. Li, and H. Zhou, "Fokker-Planck equations for a free energy functional or Markov process on a graph, Arch. Rational Mech. Anal., 203(3), Pages: 969-1008 (2012). 
[11] N. Gigli, J. Maas, "Gromov-Hausdorff convergence of discrete transportation metrics", SIAM Journal of Mathematical Analysis, 45(2), Pages 879-899 (2013).

[12] R. Eymard, T. Gallouet and R. Herbin, "The finite volume method", Handbook of Numerical Analysis. Vol. VII, North Holland, Amsterdam, Pages 713-1022 (2000).

[13] A. Mielke, "Geodesic convexity of the relative entropy in reversible Markov chains", Calc. Var. Partial Differential Equations, 48(1), Pages: 1-31 (2013).

[14] M. Erbar, J. Maas "Gradient flow structures for discrete porous medium equations", Discrete and Continuous Dynamical Systems, 34(4), Pages 1355-1374 (2014). 